\title{
POLÍTICAS URBANAS Y SOSTENIBILIDAD EN CHINA
}

\author{
URBAN POLICIES AND SUSTAINABILITY IN CHINA
}

\author{
Aida Anguiano de Miguel \\ Asociación Castellano-Manchega de Sociología (Toledo). España/Spain \\ aida.anguiano@gmail.com
}

Recibido/Received: 07/06/2016

Modificado/Modified: 3/08/2016

Aceptado/Accepted: 6/09/2016

\section{RESUMEN}

Pretendo analizar la reciprocidad entre políticas urbanas y crecimiento económico; y el papel de agentes y promotores en China. Economía emergente con altos índices de desigualdad. El funcionamiento de las empresas capitalistas y el papel del Estado en la construcción y reconstrucción de las ciudades. Examinar las infraestructuras, promotores de negocios y comercio, espacios deportivos y de ocio, centros académicos y culturales, y vivienda en las metrópolis de China. La rápida urbanización, la industria, y las infraestructuras, han convertido el país asiático en uno de los más contaminantes. Equipos de arquitectos han proyectado edificios sostenibles, pero las megalópolis hacen necesarias las ecociudades.

\section{PALABRAS CLAVE}

Políticas urbanas, economía, infraestructuras, sostenibilidad, ecociudades.

\section{SUMARIO}

1. Introducción. 2. Infraestructuras en ciudades difusas. 3. Promotores de negocios y comercio. 4. Espacios deportivos y de ocio. 5 Centros académicos y culturales. 6. Vivienda.7. Impacto ambiental y sostenibilidad. 8. Eco-ciudades. 9. Conclusiones. Bibliografía.

\begin{abstract}
I mean to analyze the reciprocity between urban policies and economic growth; and the role of agents and estate developers in China. Emergent economy with high inequality rates. The roles of capitalist corporations and the Government in the construction and reconstruction of cities, Examine the infrastructures, business and commerce developers, sport and leisure areas, academic and cultural centers, and housing in the Chinese metropolis. The Asian country has become one of the most polluting ones as result of the fast urbanization, and the rapid growth of industry and infrastructures. Teams of architects have planned sustainable building but megalopoleis make eco-cities necessary.
\end{abstract}

\section{KEYWORDS}

Urban policies, economy, infrastructures, sustainability, eco-cities.

\section{CONTENTS}

1. Introduction. 2. Infrastructures in defuse cities. 3. Business and commerce developers. 4. Sports and leisure centers. 5. Academic and cultural centers. 6. Housing. 7. Environmental impact and sustainable project. 8. Eco-cities, 9. Conclusions. References. 


\section{INTRODUCCIÓN}

El Fondo Monetario Internacional (FMI) informó que en el inicio del siglo XXI las inversiones financieras mundiales crecieron notablemente. Hecho que coincidió con una intensa concentración de activos financieros transnacionales que, procedentes de fuera del país de origen del capital, creció 2,6 veces entre 2000 y 2012 (...). El crecimiento de capitales foráneos en los mercados financieros pasó el 5\% en la década de 1960 hasta el 47\% y el 78\% en 2001 y 2012, respectivamente (Brunet Icart, 2016: 52).

"Lo económico, con la convención social del dinero y sus afanes de crecimiento permanente con evidente incidencia en el territorio, el urbanismo y la construcción". Empeño enfermizo del crecimiento económico hace que, con los potentes medios técnicos disponibles, la especie humana aparezca como una especie de patología terrestre (Naredo, 2005: 59).

En China, situada en el índice de Gini, entre 2005-2011, en 49-54 \%, el $20 \%$ más rico gana 12 veces con respecto al $20 \%$ más pobre. El aumento de concentración de riqueza de los millonarios entre 1986 y 2005 es del 140\%, los salarios de los altos ejecutivos adquieren niveles récord con participación de beneficios y acciones. Son ejecutivos de grandes compañías. Y la tendencia al aumento de la desigualdad a partir de 1990 es patente (Miguel, 2014: 351).

La construcción de la ciudad es un proceso conflictivo entre agricultor y urbanita, entre propietarios pequeños y grandes, entre propietarios del suelo y promotores urbanos, entre empresarios industriales y promotores inmobiliarios, entre promotores de viviendas populares y los promotores de viviendas de lujo, que desean mantener alejadas las viviendas obreras. Conflictos entre agentes privados y públicos, entre los propietarios del suelo y los ayuntamientos o la administración del Estado. Los agentes públicos pueden buscar la mejora de la calidad de vida, el bienestar social, la preservación de los valores ambientales o estéticos; los privados su beneficio económico, perseguir el poder político o el prestigio social (Capel, 2013:18-19).

El crecimiento acelerado de las ciudades chinas durante los últimos treinta años ha provocado dramáticos cambios en su morfología. Lo que antes eran pequeños callejones, ahora son enormes avenidas. Los antiguos mercados al aire libre, han sido reemplazados por centros comerciales, provocando atascos de bicicletas en filas interminables. Cada ciudad tiene elementos arquitectónicos y de paisaje que las diferencia, pero cada vez es más complicado encontrar esta identidad en las ciudades chinas por culpa del proceso intensivo de urbanización.

La apertura económica de China desde los primeros años ochenta del siglo pasado ha hecho que posea las más modernas tecnologías en el sector del vidrio, el acero y la piedra. Existe una nueva generación de arquitectos influidos por los estudios de arquitectura internacionales establecidos en China. Reestructuración completa de las ciudades a escala urbanística y arquitectónica. En los últimos 30 años, el país asiático ha incorporado muchos de los parámetros occidentales y sus fórmulas de progreso y desarrollo.

En 2009, China representó casi el $43 \%$ de las ventas mundiales de maquinaria de construcción. Se han construido rascacielos los más altos del mundo. Hay más de 5000 oficinas de arquitectos (30\% estatales); 150 de los 200 estudios de arquitectura más grandes del mundo, están en este país. El papel de las empresas constructoras se ha centrado en las megalópolis. 


\section{INFRAESTRUCTURAS EN CIUDADES DIFUSAS}

El desarrollo de infraestructuras en China ha sido vertiginoso a partir de la reforma económica de Deng Xiaoping (1978), consolidada a principios de los noventa, ha situado a China en la tercera posición mundial en términos económicos y primera posición como potencia exportadora. En sólo 20 años han emigrado del medio rural al urbano 400 millones de habitantes y se espera un trasvase de población hacia las ciudades de 300 millones en los próximos 20 años. A finales de los años setenta, la población urbana tan sólo era el 20\%; en 2011, la mitad de la población china era urbanita; y en 2030, el porcentaje de población urbana rondará el $70 \%$.

\subsection{La Red ferroviaria de alta velocidad, la CRH (China Railway High Speed)}

La planificación de la red se inició en la década de 1990, cuando el Ministerio de Ferrocarriles presentó una propuesta para construir un ferrocarril de alta velocidad entre Beijing y Shanghai. Ofrece líneas de alta velocidad desde el 18 de abril de 2008; el 1 de agosto con la línea de alta velocidad Beijing-Tianjin; desde 2009, ha renovado líneas antiguas para velocidades de $200 \mathrm{~km} / \mathrm{h}$ y uso mixto de viajeros / mercancías: $2.902 \mathrm{~km}$ a 31 de diciembre de 2010; y el 31 de diciembre de 2015, tiene la red de alta velocidad más grande del mundo, formada por $9.870 \mathrm{~km}$ para $300 \mathrm{~km} / \mathrm{h}$ o más y $8.931 \mathrm{~km}$ de líneas nuevas para $200 / 250 \mathrm{~km} / \mathrm{h}$. En total suman $18.801 \mathrm{~km}$ con la mayor velocidad comercial del mundo, con $350 \mathrm{~km} / \mathrm{h}$. Tiene la red de alta velocidad más utilizada del mundo, tras desbancar a Japón en 2011, con 530 millones de viajeros. En febrero de éste año, el ministro de ferrocarriles fue acusado de corrupción.

Las preocupaciones sobre la seguridad, los altos precios de los billetes producían escasos viajeros, y el impacto ambiental debido al alto consumo de energía. En mayo de 2011, el Ministerio de Protección del Medio Ambiente, ordenó la paralización de dos líneas de alta velocidad.

\subsection{Aeropuertos}

China tiene el segundo y tercer aeropuerto que mueven más cargas del mundo; y el puerto de Shanghai es el que transporta más contenedores. La circulación de personas y mercancías está fragmentada y canalizada hacia los espacios que generan más valor en términos de capital. Los aeropuertos conectan ciudades de todo el mundo.

En 1997 Hong Kohn dejaba de ser gobernada por Inglaterra y pasaba al gobierno chino. Ante la saturación del antiguo aeropuerto, con una única pista de aterrizaje, necesitaba maximizar su comercio, su principal actividad y mayor flujo de pasajeros. El Aeropuerto Internacional de Hong Kong, de Foster \& Partners, 1992-1998, es otra megaestructura, conocido como Shek Lop Kok construido en la isla del mismo nombre, inició sus operaciones comerciales en 1998 en sustitución del antiguo aeropuerto Kai Tak. Una infraestructura colosal, formada por otras dos islas más pequeñas, Chek Lap Kok y Lam Chan, unidas para dar lugar al área donde se ubica la megaestructura, y se ganó terreno al mar. Las montañas de las islas naturales fueron demolidas para obtener la tierra necesaria y la necesidad de puentes que debían resistir los habituales tifones y ser suficientemente altos para no restringir el tráfico descomunal de embarcaciones que llegan al puerto de la ciudad (Foster, 2005: 64).

Se tardó seis años en realizar esta obra de ingeniería, costó veinte billones de dólares. Funciona las veinticuatro horas del día, siendo uno de los aeropuertos más activos del mundo en circulación de pasajeros y de carga. Es el punto de carga más importante de Asia y el 
segundo del mundo - en 2009, tuvo cuarenta y seis millones de pasajeros y 3,8 millones de toneladas de carga (Sánchez Mosquete, 2010).

Pudong es la más importante base de tráfico de carga del mundo. El Aeropuerto Internacional de Hongqiao era el principal aeropuerto de Shanghai. Durante los años 1990, la ampliación de Hongqiao era imposible debido al desarrollo urbano. El Aeropuerto Internacional Pudong-Shanghai, con 400 millones de dólares de la ODA de Japón, se inauguró el 1 de octubre de 1999. La primera fase comenzó en 1997, tardó sólo dos años en construirse y costó 1670 millones de dólares. Ocupa un espacio de 40 kilómetros cuadrados y se encuentra a 30 kilómetros de la megalópoli; la segunda y tercera fase se inauguraron en marzo de 2005 y el 26 de marzo de 2008, respectivamente; el proyecto de la cuarta pista y la ampliación de la quinta con su aeropuerto: $30,5 \mathrm{~km}$ a una velocidad máxima de $430 \mathrm{~km} / \mathrm{h}$ desde marzo de 2004, se concluyó en 2015. El tren MagLevi es propiedad del gobierno de la ciudad, un tren de levitación magnética con tecnología Transrapid, único en el mundo, une la ciudad con el aeropuerto.

El primer Aeropuerto de Pekín fue inaugurado en 1958 por La República Popular China. Se trataba de un pequeño edificio terminal; en 1980 fue inaugurado un edificio más grande con muelles para 10 o 12 aviones; y en 1999 fue construida la Terminal 2. La nueva Terminal Internacional y el Centro de Transporte Terrestre (CTT), 2003-2008, abarca una superficie de 1,3 millones de metros cuadrados. Es la megaestructura más grande y avanzada por su tecnología, pero también por eficacia operativa y sostenibilidad. Obra de Foster and Partners e Ingeniería Arup. Cubiertas aerodinámica que evoca el vuelo y forma de dragón, símbolo tradicional chino. Un proyecto sostenible: una única marquesina fuente de luz natural; lucernario orientado al sudoeste, que maximiza el calor y sistema integral de aprovechamiento de recursos. Los materiales se seleccionaron en función de su disponibilidad local y se construyó en cuatro años. Patrocinado por Beijing Capital International Airport Company Ltd., situado a 32 kilómetros al noreste de Pekín y a 19,5 kilómetros de las Villa Olímpica. Es la segunda Terminal más grande del mundo por detrás de la Terminal de Dubái. Proyecto innovador en términos de eficacia operativa, confort de pasajeros y sostenibilidad.

El aeropuerto comprende tres cuerpos alargados, dispuestos siguiendo una línea longitudinal, en que los dos extremos se abren a modo de abanico para situar los vestíbulos de salidas y llegadas. El edificio del lado sur, abraza un jardín ovalado, en cuyo centro se sitúa un cuarto volumen con forma de avellana, el GTC (Ground Transportation Centre). La longitud de norte a sur es mayor de tres kilómetros, las tres piezas se mantienen conectadas visualmente. En el eje central, se sitúa un patio flanqueado por una secuencia de pilares rojos, evocando los templos chinos. La cubierta está formada por una estructura tridimensional de acero, revestida por una chapa metálica coloreada, en la que se recortan los lucernarios triangulares. La curva, se eleva en el punto medio para crear un espacio central catedralicio. El edifico incorpora numerosos sistemas pasivos de eficacia energética, que minimiza la energía consumida (Foster, 2013: 286-293).

Aeropuerto Internacional Shenzhen Bao, Terminal 3, por el rápido desarrollo de la ciudad, los arquitectos Maximiliano y Doriana Fukgas, ganaron el Concurso Internacional. Construido en tres años. El promotor es Shenzhen Airport Group Co. El edificio evoca la imagen de una manta raya. De 1,5 kilómetros de largo, con luces de techo de hasta 80 metros y paneles de metal y vidrio en forma de nido de abeja en la fachada, que permite que la luz natural se filtre al interior y apoyos de columnas cónicas blancas, en una escala de catedral (Lalueta, 2013). El mismo estudio de Arquitectura participará en las dos fases de ampliación, programadas para finalizar en 2025 y 2035 , respectivamente. 


\subsection{La Red Nacional de Autopistas}

Esta red conecta las grandes metrópolis, pero otras partes de las ciudades y las zonas rurales quedan al margen de las autopistas. Los lugares que se conectan son relevantes en la circulación de capital. Las autopistas urbanas crean fragmentos espaciales intraurbanos, que separan y excluyen zonas de la ciudad, crean marginación y aislamiento y rompen la continuidad urbana.

El gobierno chino ha propiciado la construcción de autopistas. La planificación es prerrogativa del Ministerio de Transportes, que aprobó el plan para la "Red Nacional de Autopistas" en 2004, y la Comisión Nacional de Desarrollo y Reforma (GPADB, 2008). Negoció con los gobiernos provinciales sobre la localización de las autopistas: un planeamiento en forma de red (siete que van desde diversos puntos de China a la capital); otros nueve que conectan de norte a sur, y 18 de este a oeste. La extensión total era de 85.000 kilómetros en 2013 (Hidalgo, 2016:7). Todas las autopistas son de peaje, por tanto no es un bien público.

\subsection{La Presa de las Tres Gargantas}

La mayor presa hidroeléctrica del mundo, situada en el curso del río Yangtse, entre las ciudades de Chongqing y Yichang, a su máxima capacidad retiene el agua a 91 metros sobre el nivel del río y contiene aproximadamente 39.300 millones de $\mathrm{m}^{3}$ de agua, en una superficie de 632 kilómetros cuadrados. Pensada para proveer el $10 \%$ de la energía eléctrica china; sin embargo, el crecimiento de la demanda ha sido mayor y sólo suministra el $3 \%$ del consumo. La propuesta para su construcción se inició en 1919, parte del "Plan Industrial", pero es en 1992 cuando se aprueba el proyecto y se acabará de construir en 2009. Inundará más de $632 \mathrm{~km}^{2}$ de tierra (incluyendo 24.500 ha de tierras cultivables), 13 ciudades, cientos de pequeñas aldeas, que afectan a un total de 1.130 .000 personas, y se perderán tesoros arqueológicos y culturales: restos del paleolítico y fósiles paleontológicos; más de 80 lugares del neolítico y unas 500 tumbas aristocráticas y 300 construcciones de las Dinastías Ming (1368-1644) y Qing (1644-1911).

Se pretende regular los aumentos de caudal para evitar las inundaciones, abastecer de agua a una gran parte de la población y permitir el mejor aprovechamiento de la navegabilidad. Se generarán hasta 84 millones de KW al año de electricidad. El templo de Zhang Fei (220-280 d.C.) se desplazará y será reconstruido en el municipio de Panshi, pero el traslado requerirá una inversión de 8,5 millones de dólares. Los problemas que la presa traerá en el medio ambiente son muy graves, así como sociales (Negre, 2007:1-3).

Impacto sobre la biodiversidad - especies vegetales y animales y efectos significativos sobre la diversidad acuática -, afectando a los peces migratorios (esturión, pez espada y el famoso delfín del Yangtzé). El vertido de sustancias tóxicas al río supone una amenaza adicional (López-Pujol, 2008: 134).

\section{PROMOTORES DE NEGOCIO Y COMERCIO}

China es el mercado de la construcción más grande del mundo y el mayor consumidor de cemento, el 56\% del total mundial. La Mega-región del Delta del río Perla, al sureste y la excolonia de Hong Kong se ha extendido hasta unirse con Shenzhen, una de las áreas económicamente más importantes. La tendencia actual de producir urbanizaciones consiste en la implantación de usos y funciones en el territorio de un modo disperso, buscando la compatibilidad entre los usos y la mejor ubicación de las actividades económicas. 
Han surgido megalópolis en constante crecimiento de personas, que conviven y se relacionan en ciudades complejas, donde la calidad de vida es distinta según los grupos sociales. Las ciudades actuales son el resultado de la globalización y las políticas neoliberales. Las reformas de 1978 optaron por la China del Sur y la macrourbanización, fueron elegidas primero las ciudades costeras, dando lugar a corredores urbanos casi ininterrumpidos, siguiendo el Gran Canal Chino Beijing -Hougzhon y los deltas de los ríos Yangtsé y Perla en el Sur. Se confirió a las ciudades capacidad de establecer contacto directo con el capital extranjero y con el local. El Estado conserva las decisiones políticas, pero las ciudades controlan las decisiones económicas (Gómez Mendoza, 2014).

\subsection{El Banco de Hong Kong y Shanghai, 1986}

Paso de China de una economía socialista a una economía de mercado. Ubicado en el área central, de Foster \& Partners, en la antigua colonia británica, de alta tecnología, es recordado por su altísimo coste. El reto era diseñar "la mejor sucursal bancaria del mundo". El proyecto analizó la naturaleza del sector bancario en Hong Kohn y la plasmó en la forma construida: $90.000 \mathrm{~m}^{2}$ y calendario ajustado requirió un alto grado de prefabricación y uso de módulos acabados en fábrica. Estructura suspendida, con pares de mástiles de acero dispuestos en tres crujías. Forma perfil escalonado de tres torres individuales de 29, 36 y 44 plantas, respectivamente, que crean plantas de anchura y profundidades distintas, y terrazas ajardinadas. Un tragaluz tubular, de espejo instalado en el tejado, refleja la luz natural a través del atrio hasta la planta baja, ocupada por una explanada pública cubierta. Desde ésta unas escaleras mecánicas ascienden al vestíbulo principal del banco, acristalado como escaparate. La circulación combina ascensores de alta velocidad, que conducen hasta las zonas de recepción, con una serie de escaleras mecánicas, que conectan los diversos grupos de plantas (Foster, 2013: 248-253).

\subsection{El distrito de Pudong en Shanghai}

Esta zona industrial se ha transformado en un ejemplo de urbanismo del siglo $\mathrm{XX}$, con rascacielos, centros comerciales, plazas, aparcamientos, hoteles. Centro internacional de negocios. Nueva área urbana, se extiende al este hacia el Mar de China oriental, sobre un land que hace pocos años estaba ocupado por barracas y ciénagas, donde los edificios no superaban las dos plantas, se ha transformado de ciudad horizontal en vertical.

En Pudong las calles son anchas y limpias distintas al centro Huangpu. Edificios rodeados por zonas verdes impracticables. El objetivo de la administración pública es desarraigar la improvisación, como los mercadillos al aire libre y las comidas en las calles, que son vistas como índices de pobreza. El puerto de Shanghai es el que mueve más contenedores del mundo.

Beijing (Pekín) ciudad antiquísima, capital durante la dinastía Yuan (1206-1368) y desde 1949, metrópoli política y cultural, y, en la década de los ochenta, transformación. El urbanismo estrecha relación con el crecimiento y expansión del sistema económico mundial, ha producido desarrollos urbanos similares. El capitalismo inmobiliario tendrá el papel primordial de organizar y equipar el espacio, para incrementar la eficiencia del capital comercial, industrial y financiero, ayudado por el Estado.

Se fomentaron las zonas comerciales de desarrollo y las empresas extranjeras de negocios, para atraer inversión privada y promover empresas mixtas, transformando el paisaje urbano. Los grupos financieros invierten en operaciones inmobiliarias, agentes urbanizadores que contribuyen a la conversión del espacio rural en espacio urbano. 
La economía política del espacio y "cómo diferentes clases de gentes y de instituciones luchan por conseguir sus diferentes objetivos en la creación de la metrópoli"; esfuerzos de las élites locales por hacer dinero a partir del desarrollo (Capel, 2006: 22). El suelo y la propiedad del suelo se convierten en una mercancía sobre la que la gente pone edificios.

\subsection{CCTV, nueva Sede de la Televisión Central de China}

Un concurso internacional ganado en 2002, por Office Metropolitan Architecture (OMA) e Ingeniería ARUP, y acabado en 2012. Estudio de arquitectura fundado por Koolhaas (1944), Premio Pritzker en el 2000. La CCTV es un rascacielos de 234 metros de altura y 44 plantas en el distrito central de negocios de Pekín. Situado junto al tercer anillo viario de la ciudad, acoge en sus $473.000 \mathrm{~m}^{2}$ todas las fases de proceso, producción y control de más de 250 canales. El complejo se completa con un centro cultural público con hotel, y el denominado Media Park, un área ajardinada para rodajes y eventos al aire libre. Distribuidas en un "bucle" de actividades interconectadas, que da forma al edificio. Desde una plataforma común, de siete plantas más tres soterradas, se elevan dos grandes pilas inclinadas seis grados, dedicada a la retransmisión y otra a servicios, investigación y educación, hasta unirse en la planta 36, generando un cuerpo en voladizo de 75 metros, que alberga las oficinas. Mediante un programa de simulación sísmica, se garantiza la resistencia del CCTV a un terremoto de grado 8 (Koolhaas, 2006: 270-311)

\subsection{TPT Three Partnership Tower, 2004}

Es una obra de Herzog \& de Meuron, Premio Pritzker 2001, situado en al noroeste de la tercera circunvalación en Pekín. Intersección de tráfico donde se reúnen grandes carreteras elevadas. Volumen poligonal que se yuxtapone a la velocidad y al flujo de carreteras, logra elevación y puntos de vistas distintos. El edificio se integra en el contexto existente y define una plaza enfrente de la edificación. La torre crece en altura y cambios de volumen, en la parte norte y, en la parte sur, el volumen se adelgaza: abre el espacio del edificio hacia el sur y lo cierra hacia el norte. Se propone utilizar este espacio como pequeño parque público con vegetación densa, que la protege del ruido de grandes carreteras. La planta baja, se convertirá en el espacio de conexión entre el parque, en la parte trasera, y la plaza delante del TPT. Tres volúmenes, de altura diferente, se funde en un sólo, lo que maximiza la penetración de la luz solar y la ventilación., y realza el aspecto escultórico del edificio: la superficie deformada se triangula y ligeramente ondulada, se divide en unidades prismáticas más pequeñas, sólo unos pocos reflejan de forma individual la luz directa del sol. La fachada se construye con un muro cortina de doble piel de vidrio, la capa externa proporciona un filtro para el ruido y la contaminación del aire, así como una barrera térmica. El color rojo caliente de la capa interna de la fachada añade profundidad espacial. Dependiendo de la hora del día y de las condiciones meteorológicas el calor del edificio cambia constantemente (Herzog \& de Meuron, 2004 ).

\subsection{CDB Distrito Comercial Central de Pekín}

Es el resultado de un Concurso Internacional al que acudió OMA. La propuesta de un núcleo para Pekín: las torres han contribuido a que los distritos comerciales y de negocios sean iguales en todas partes. El núcleo del CBD debe definirse como una tipología que aliente la interacción humana y la comunicación: tendrá unas 300 torres; evolucionó para convertirse en una red de núcleos dispersos, de baja altura y con áreas flexibles de oficinas, en combinación con espacios comerciales y recreativos, que no sólo extrema la posibilidades 
de contacto, sino que ofrece la oportunidad de que el CBD tenga vida urbana las 24 horas del día (Koolhaas, 2007:178-187).

Shenzhen es el mejor ejemplo de la conversión de China del comunismo al capitalismo. El Gobierno implicó a jóvenes arquitectos chinos para convertir este pueblo de pescadores en "zona económica especial". En la actualidad es un gigantesco centro tecnológico y una de las ciudades que más rápidamente está creciendo en el mundo. Centro financiero y comercial, ciudad de rascacielos donde viven y trabajan 15 millones de habitantes.

\subsection{La Compañía de Energía Shenzhen}

Para esta ocasión se convocó un concurso internacional para la edificación de la Shenzhen Energy Mansion, en 2009. El proyecto ganador fue del estudio BIG-Bjarke Ingels Group, fundado en 2006, por el joven arquitecto danés Bjarke Ingels (1974). La propuesta del estudio BIG, es una torre, que en apariencia es una sutil mutación del rascacielos clásico, pero de bajo consumo energético (Anguiano, 2015: 237).

\subsection{Shenzhen Stock Exchange}

Es una obra de OMA, acabada a inicios de 2014. Una caja elevada de tres alturas desarrolla actividades del mercado bursátil, de $15.000 \mathrm{~m}^{2}$ por planta, a 36 metros de nivel del suelo, libera la planta baja y crea una plaza pública cubierta, cedida a la ciudad. La cubierta de la caja cuenta con un jardín vegetal para empleados y usuarios. La actividad bursátil y comercial tiene lugar en el podio; en los pisos superiores se ubican las oficinas privadas de la Bolsa, y en los niveles más altos oficinas para alquilar y un restaurante. La fachada de vidrio hace visible a la ciudad las actividades bursátiles. El color neutro de la fachada cambia según las condiciones climáticas (Koolhaas, 2015:186-189). Diseñado para ser un edificio verde, clasificado con tres estrellas en China.

\section{PROMOTORES DE ESPACIOS DE DEPORTE Y OCIO}

Los establecimientos de la Villa Olímpica han elevado los costes de las propiedades del área. Lo directores financieros buscaban que las Olimpiadas sean comercialmente viables. Discrepancias entre los prestigiosos estudios de arquitectura que han ganado los concursos internacionales y la mano de obra china y la calidad de los materiales; sin embargo una regulación específica que los estudios de arquitectura extranjeros deben trabajar con un socio de China, lo que ha promovido una nueva generación cosmopolita de arquitectos chinos (Amiano, 2009: 8).

\subsection{Estadio de Beijing}

Megaestructuras sostenibles fueron realizadas a través de concursos internacionales para las Olimpiadas en el distrito de Chaoyang, en Pekín. Los promotores urbanos en la construcción del Estadio de Beijing fueron el Consorcio CITIC (China International Trust and Investment Corporation), afrontó el $42 \%$ de la financiación total del proyecto a cambio de una oferta de utilización del estadio por un periodo de 35 años una vez finalizados los Juegos Olímpicos. El Consorcio CITIC es una empresa de inversión de la República Popular China, fundada en 1979, en Pekín. Su objetivo era atraer y utilizar capital extranjero, en la actualidad tiene principalmente bancos en China y países desarrollados. En 2006 el BBVA acuerda con el grupo bancario CITIC adquirir un 5\% en CNCB por 501 millones y un 15\% 
en CIFH por 488 millones; y en 2013 y marzo 2016 ha decidido vender y los beneficios han superado los que se esperaba lograr en la inversión.

Colaboraron el "Grupo de Construcción Urbana de Beijing"; el "Grupo Golden State" de los Estados Unidos, y la filial "Guoan Elstrong", bajo un acuerdo asociativo entre el sector público y privado. El 58\% del presupuesto restante fue financiado por el Gobierno Municipal de Beijing.

El estadio fue diseñado por los arquitectos suizos Herzog \& de Meuron, ganadores del Concurso Internacional convocado en 2003. En la construcción colaboró el Grupo de Investigación de Arquitectura y Diseño Chino. Los arquitectos se plantearon proyectar una megaestructura que siguiera siendo funcional después de los Juegos Olímpicos de 2008, con un volumen de tres millones de $\mathrm{m}^{3}$, considerado el espacio cerrado más grande del mundo y la estructura más baja con 26 kilómetros de acero; sostenible: en invierno, el sistema absorbe el calor del suelo, lo que ayuda a calentar el estadio, mientras que en verano, el frío del suelo, se acumula para refrescarlo. Diseñado para soportar terremotos ya que está situado en una de las zonas más sísmicas del mundo. (Anguiano, 2015: 238-239). "Hoy el estadio es un museo, ya no se utiliza. La monumental construcción que costó 480 millones de dólares y cuesta alrededor de 11 millones cada año de mantenimiento, no tiene inquilino" (Arkiplus, 2016).

\subsection{El Olimpic Aquatics Center}

El conocido como "El Cubo de Agua", proyectado por los australianos PTW, dirigido por el arquitecto John Pauline, forma un conjunto con el Estadio Olímpico. En julio de 2003, este estudio, junto a Ingeniería Arup, la Corporación Estatal de Construcción e Ingeniería China (CSCEC) y el Instituto de Diseño de Shenzhen, ganaron la licitación internacional para diseñarlo. Una estructura rectangular, cubierta por unas singulares membranas plásticas poliédricas que permiten el paso de la luz natural. Ha costado 75 millones de euros, aportado por donaciones de chinos residentes en el extranjero. Se accede a través de puentes y está rodeado de un foso con agua. Capacidad para 17.000 espectadores y 11.000 asientos extras, que se retiraron una vez finalizados los Juegos. Se utilizaron 6700 toneladas de acero y 1300 toneladas de varillas para la construcción sobre una superficie de $80.000 \mathrm{~m}^{2}$. La mayor estructura del mundo recubierta, con más de $100.000 \mathrm{~m}^{2}$ de cojines de ETFE (burbujas de plástico, que permite más entrada de luz y calor que el cristal, con una disminución de $30 \%$ de gastos de energía). Cada año la superficie exterior y el techo pueden colectar 10.000 toneladas de lluvia, en tanques subterráneos para usar en piscinas una vez reciclada; 70.000 de agua limpia y 60.000 toneladas de agua de piscina; además de 140.000 toneladas de agua reciclada por año. Aprovecha la energía solar para calentar el agua de las piscinas. Los materiales y el diseño permiten soportar movimientos sísmicos (Pauline, 2014). "El Cubo del Agua" ha sido renovado como parque temático. Se han invertido 50 millones de dólares: una piscina climatizada, río lento, 13 toboganes, que ocupan alrededor de la mitad del complejo.

\subsection{Centro Comercial, cultural y recreativo, en el barrio de Jindong, Jinhua, 2003}

Este centro es un proyecto urbano de $270.000 \mathrm{~m}^{2}$, constituido por un conjunto de tiendas, restaurantes, hoteles, oficinas y apartamentos, localizados, en el área de nuevo crecimiento de Jinhua, una ciudad de cuatro millones de habitantes, de la provincia de Zheijang. La municipalidad de la ciudad conocieron el estudio de Herzog \& Meuron a través de Ai Weiwei, una figura de referencia del arte chino contemporáneo (su padre, el conocido poeta Ai Quing). El Ayuntamiento encargo a Ai Weiwei que diseñara el esquema general de un parque cultural en memoria de su padre, persuadió a las autoridades municipales para que solicitaran una propuesta a nuestro estudio. Una solución de diseño integral aparecía como lo 
más deseable. El programa prevé la creación, en plazo de dos años, de un centro urbano capaz de acoger a 100.000 habitantes, y en los años siguientes pueda convertirse en el principal foco comercial y cultural para una población de 300.000. Los campos de cultivo de arroz son el punto de partida. Concentración del alojamiento en el extremo norte para dejar al sur un parque, superpusimos otra malla sobre el terreno (Hergoz \& Meuron, 2006: 366-373).

\section{CENTROS ACADÉMICOS Y ESPACIOS CULTURALES}

5.1. Tree Village Campus, Pekín, 2003-2004. Esta obra de Hergoz \& de Meuron, es un proyecto puesto en marcha por un inversor privado, un nuevo modelo de campus en el que la educación de adultos se combine con el ocio, la cultura y el comercio. El solar con una superficie de $350.000 \mathrm{~m}^{2}$, en un lugar estratégico del distrito Noroeste de Haidian, en Pekín, situado sobre el quinto anillo y próximo a las universidades. El diseño fusiona las cualidades y características de la retícula urbana de Pekín, con sus casas patio y los recorridos en zig-zag de los jardines chinos tradicionales. Los bloques se entrelazan de forma infinita, lo que produce un lenguaje diverso de patios, espacio, ejes de circulación peatonal, plazas y pequeños parques. Las bandas dan una enorme flexibilidad en cuanto a zonificación y posibilidades de adaptación a las necesidades de los usuarios. El mismo motivo se aplica a diferentes escalas, a bloques, estructuras, acabados de fachada, protección solar y pavimentos (Herzog \& de Meuron, 2006: 166-173).

5.2. Campus de la Universidad Chu Hai, en Hong Kong, de OMA, 2009, $28.000 \mathrm{~m}^{2}$, sobre una colina con vistas sobre la bahía de Castle Peak, el nuevo campus acoge tres facultades, dos centros de investigación, alojamiento para el personal, espacio para interrelación de estudiantes y personal de la universidad, además de biblioteca, cafetería, gimnasio y aulas magnas. Previsión de dos años para su construcción que determina el uso de elementos estructurales sencillos y de rápida ejecución. Dos bloques paralelos de ocho alturas, entre las cuales se dispone una base común con plataformas y escaleras, sigue la pendiente del terreno hacia el mar y comunica los niveles inferiores de ambas piezas. Bajo este plano escalonado, se despliega una base común que une los cuatro niveles inferiores de los bloques, y que alberga el programa multidisciplinar (Koolhaas, 2015: 186-189).

5.3. Campus de la Universidad de Arte, Hangzhou. El arquitecto chino Wang Shu (1955), premio Pritzker 2012, fundador de Amateur Architecture Studio, es autor del Nuevo Campus de la Universidad de Arte, 2002-2007, en Hangzhou. Complejo de pabellones, dormitorios, aulas y edificios administrativos, bien articulados y proporción entre volúmenes y espacios libres. Ha utilizado materiales disponibles en la zona, mano de obra cualificada y presupuestos ajustados. Plantea un entorno arquitectónico de calidad, a través de una síntesis de las tradiciones locales y las soluciones globales. Relación con el "lugar".

5.4. Casa del Libro, Pekín, 2003, Concurso Internacional ganado por OMA. Será la librería más grande del mundo. Para regular la presión que supone recibir más de 50.000 visitantes diarios, el nuevo edificio cuenta con un sistema de rampas que se conectan con lo existente, mezclando ambas partes en una única planta continua. Las zonas de venta crean un movimiento espiral de estanterías en suave pendiente, que acogen y guían el movimiento de los clientes. La fachada se convierte en una gigantesca estantería translúcida. Dos generosas aberturas, hacia la avenida Chang An y la plaza Xidan, articulan las entradas principales y 
forman monumentales pantallas electrónicas, que emiten cara a la ciudad lo que acontece en la librería y hacia el interior información del resto del mundo (Koolhaas, 2007: 30-37).

5.5. Museo de Arte Contemporáneo de Shanghai (MOCA), 2005, del estudio Lin Yuyanes, con sede en Hong Kong y Shanghai, ubicado en el parque del Pueblo. Primera institución sin ánimo de lucro e independiente. Estructura acristalada, En la tercera planta restaurante y los llamados Art Lab, cócteles, exquisitos platos con música, con una azotea con vistas a la ciudad. El museo organiza lecturas, debates y encuentros creativos. Durante las tardes de fin de semana, profesores de arte realizan actividades en el Kid's Corner; y los adultos disponen de clases de historia e iniciación al arte, así como talleres prácticos. Ofrece tarifas de entrada con descuento para estudiantes, jubilados y grupos. El parque está bien comunicado mediante transporte público (Lin Yuyanes, 2005).

5.6. Plan director del West Kowloon Cultural District, Hong Kong (2009), de Foster \& Partners, en un terreno de cuarenta hectáreas, el barrio cultural se convertirá en uno de los focos artísticos más grandes del mundo, incluye 17 centros culturales que acogerán todo tipo de espectáculos, desde teatro tradicional chino hasta conciertos de música Pop: 30.000 metros cuadrados de equipamientos educativos, fomentarán el talento musical y asegurarán la rentabilidad del distrito a largo plazo. El barrio de usos mixtos combina la vivienda y el lugar de trabajo con galerías, estudios, tiendas, cafeterías y restaurantes, integrando los centros culturales en la vida diaria de la ciudad.

El barrio incluye un parque público de 23 hectáreas: una serie de terrazas al aire libre y senderos arbolados conectarán los edificios culturales con el frente marítimo. El equilibrio entre coches y peatones se ha conseguido situando la ruta principal de vehículos bajo el nivel del suelo. El parque se ha diseñado para conseguir un balance neutro de emisiones de carbono, y se han proyectado sistemas de alta eficacia e infraestructuras de bajo consumo. El proyecto energético incluye una instalación global de climatización, el reciclaje de aguas grises, un sistema de recuperación energética a partir de aguas residuales y la generación de electricidad limpia (Foster, 2013: 96-99).

5.7. Entre los equipamientos culturales destacan la Great Opera House, Guangzhon, 2010, construido por la arquitecta Zaha Hadid, en en colaboración con ingeniería Arup. Apertura hacia el río Pearl. El Auditorio para 1800 asientos, con la más alta tecnología acústica y la sala multifuncional con capacidad para 400 espectadores, para muestras de arte, opera y conciertos: 70.000 metros cuadrados. Situando el auditorio en los niveles superiores y enterrando el centro de exposiciones, funciona durante el día, el auditorio acogerá eventos por la noche, creando un centro con actividad las 24 horas. Su diseño tuvo influjos de los valles fluviales y la forma en que éstos erosionan” (Zaha Hadid, 2011).

5.8. Museo nacional de las artes de China (Namoc), proyecto de Jean Nouvel en colaboración con el Beijing Institute of Architectural Design. El más grande museo construido en Asia $\left(130.000 \mathrm{~m}^{2}\right)$. El Namoc destaca por la riqueza de sus colecciones. Un volumen de piedra, cristal y acero de más de 200 metros de largo acogerá exposiciones permanentes y temporales del arte Ming (1368-1644); jardín interior encargado al paisajista Gilles Clément. Ubicado en el eje histórico de Pekín, enfrente del estadio Olímpico, deberá abrir sus puertas en 2017 (Nouvel, 2014:16-17). 
5.9. Complejo Cultural, Longgang, subprovincia de Shenzhen, diseñado por Mecanoo, en colaboración con Ingeniería Grontmij. El Concurso internacional de ideas para $90.000 \mathrm{~m}^{2}$, ganado por el estudio dirigido por Houben en 2011, iniciada la construcción en 2014, prevista para inaugurarse en 2015, después se pospone a 2017 y en la actualidad al 2018. El complejo edificado conectará el parque con nuevas áreas residenciales.

Tiene como objetivo convertirse en un punto de referencia y transformar el parque Longchen en un lugar de esparcimiento. Cuatro volúmenes forman curvas y se elevan en diferentes direcciones y los arcos permiten el flujo de peatones y extender la edificación, en la planta baja, hacia los espacios libres, conectando con la ciudad. Desde el interior de la edificación se extienden dos puentes que conectan los locales comerciales con la nueva zona residencial. La morfología genera grandes arcos, que crean amplios espacios para el tránsito peatonal, entre ambas zonas.

Los promotores Longgang Government y Vanke en Book City, pretendían unir el centro financiero con el cultural. Un proyecto faraónico, de un programa variado: Centro comercial del Libro, de $22.500 \mathrm{~m}^{2}$, Museo de Arte, Centro Juvenil y Centro de Ciencias. De 5 plantas de altura y aparcamientos subterráneos, y una plaza pública de alrededor de $70.000 \mathrm{~m}^{2}$. Fachada roja brillante. Un conjunto sostenible, de volúmenes con aislamiento térmico en su envolvente y elementos translucidos para buena iluminación natural; el agua de lluvia se recogerá para reutilizarla en la refrigeración y en los lavabos, y un intercambiador de calor (aire) (Lalueta, 2014).

\section{DESIGUALDAD SOCIAL Y VIVIENDA}

En 1980 aparecen las primeras iniciativas de promotores privados de viviendas. Se inicia en Shenzhen la promoción de residencias para extranjeros y empleados de empresas no estatales. En 1988, la reforma constitucional mantiene la propiedad de la tierra en manos del Estado, pero permitía a los individuos comprar el usufructo de la tierra por 70 años. En 1998, el gobierno chino liberalizó el mercado de la vivienda mediante el Programa Nacional de Viviendas y fomentó la adquisición de residencias privadas, frente a las de protección oficial. De 1998 a 2005 convertirá en propietarios de las viviendas a los inquilinos.

Dificultad de acceso a la vivienda sobre todo para el trabajador migrante por culpa del sistema de propiedad chino, sólo el $0,6 \%$ de los trabajadores que emigran a la ciudad tienen vivienda propia. En 2012, más de la mitad de los emigrantes rurales son ilegales, transeúntes, población flotante. Urbanizaciones burguesas cerradas conviven con grandes barrios de infraviviendas de inmigrantes.

Shanghai ha crecido en los últimos diez años a una hora anual de alrededor del $14 \%$ y en cada año han sido demolidos edificios por una extensión de 1,6 millones de $\mathrm{m}^{2}$. Cuatro millones de inmigrantes ilegales trabajan en este metrópoli, generando problemas pero permitiendo al mismo tiempo que la economía siga creciendo (Gutiérrez, 2005:1).

Inversiones extranjeras y expatriados occidentales encontramos en el mercado inmobiliario sobre todo en ciudades costeras, como Shanghai, desgravaciones fiscales y acceso privilegiado a la vivienda. Con la apertura a la inversión privada, los ratios de metros cuadrado per cápita, equiparable a los de Europa, suben a 15 o $20 \mathrm{~m}^{2}$ (antes del 1978 era de 4 $\mathrm{m}^{2}$ ). 


\section{1. "Una ciudad, Nueve Pueblos"}

Es un ambicioso Plan urbanístico de 2001, promovido por el alcalde de Shanghai, Chen Liangyu. La idea era descongestionar la enorme conurbación para dar a sus más de 23 millones de habitantes, la opción de vivir en suburbios en el campo. Se crearon nueve diferentes urbanizaciones, dotadas de servicios, con parques, lagos y toda clase de equipamientos culturales y deportivos, intercalados con polígonos industriales, que darían trabajo a los habitantes. Las nuevas urbanizaciones serían temáticas, cada una diseñada por un arquitecto de un país diferente. Nacieron lugares peculiares: Pueblo austriaco de Hallstatt, Thames Town, una copia de la vieja ciudad inglesa, sin casi habitantes; en Baoshan se creó un poblado escandinavo y en el de Minhang una copia de una villa italiana; en Gaogiao se construyó una nueva Holanda, falsa y vacía de residentes; en el distrito de Jiading, arquitectos alemanes diseñaron una ciudad teutónica, junto a una fábrica de Volkswagen, pero casi no tiene habitantes. Las urbanizaciones que más éxito han tenido han sido la Thames Town y la falsa Alemania, las viviendas adquieren precios como en Shanghai. Asimismo, se proyectó una ciudad canadiense, obra de estilo estadounidense, pero ecológica.

El Pueblo Español sería el décimo. En el 2003 empezaron las obras cerca del centro de Fengcheng, a 60 kilómetros de Shanghai, promovidas por el más rico de esta ciudad, Zhon Zhengyi. En casi dos kilómetros cuadrados se edificaron viviendas de estilo español, color crema, tejados inclinados y tejas rojas. Fueron diseñadas por el arquitecto Marciá Codinachs. La entrada estaba decorada con molinos de viento manchegos. Había bulevares y plazas, centros comerciales, un museo y un parque español y hasta estaba previsto un teatro de la ópera. No se vendieron muchos apartamentos.

Una ciudad, Nueve Pueblos, se detuvo en 2004 cuando Zhon Zhengyi acabó procesado por corrupción. El pueblo español recuperó la inversión en 2007 y se terminaron las obras pero no se resolvieron las malas comunicaciones públicas con Shanghai. Los promotores inmobiliarios que debían adquirir los edificios para revenderlos, se quejaban de que eran pequeños, sólo de 10 metros de altura, contenían pocos pisos y pocos beneficios. En 2012, el centro comercial estaba casi vacío y las últimas viviendas a precio de saldo. El Pueblo Español es un fracaso (Cervera, 2013).

\subsection{El barrio de Hutong}

Este barrio disfruta de unos populares callejones de Pekin donde conviven de modo fraterno los vecinos y se puede contar con un mínimo espacio de tierra. Son construcciones de madera y ladrillo, de tránsito peatonal y vehículos de dos ruedas. A sus lados se suceden viviendas de $50 \mathrm{~m}^{2}$ máximo, donde llegan a vivir ocho personas, que no coinciden por sus horarios de trabajo en la vivienda en las mismas horas. Se dedican a la limpieza del callejón, deben acercar la basura hasta la avenida, donde pasa el recolector que recoge sus desechos y los lleva a la planta procesadora de residuos. Próximas a las viviendas hay quintas comunitarias de verduras, que constituyen la alimentación básica. En la periferia, se ubican conjuntos habitacionales construidos o en construcción, a partir de bloques de viviendas que superan las 4 ó 5 plantas usuales (Amiano, 2009: 6).

\subsection{Conjunto WenChengJie}

Este conjunto está situado en el noreste de Pekín y es una construcción en altura pero un espacio activo por la fusión de usos distintos. El perímetro exterior está compuesto por establecimientos comerciales (tiendas de frutas y verduras, cafés, papelerías, almacenes de gafas, etc.). Veinte establecimientos distintos y varios de ellos permanecen abiertos 24 horas diarias, lo que proporciona mayor seguridad a los habitantes. Un espacio parecido a un 
parque o jardín, con una especie de cerca que separa el espacio interior del exterior destinado a diferentes actividades. (niños y ancianos lo utilizan a lo largo del día para bailar y hacer ejercicio). Una megaestructura acogedora para personas de todas las edades, que promueve los intercambios y la comunicación de una comunidad. "El buen diseño urbano se mide por la capacidad de ser aprovechado por niños y ancianos" (Laserna, 2014).

El Gobierno chino adquiere promociones privadas para incluirlas en el parque público de viviendas. Descarga a los promotores y propicia hogares a los ciudadanos con menos recursos. El país arrastra una superficie residencial sin vender y las viviendas vacías de nueva construcción han aumentado un $190 \%$, en cuatro años. Según los datos de la Oficina Estadística China correspondientes al mes de mayo, el país cuenta con $657 \mathrm{Km}^{2}$ de promociones residenciales sin vender (Ollero, 2015).

El 12 Plan Quinquenal plantea el control de precios de la vivienda en las zonas urbanas y prevé la construcción de 36 millones de unidades de vivienda de renta baja. Esta situación afecta severamente a los habitantes de ingresos medios-bajos. El presidente del gobierno chino, Hu Jintao instó a los gobiernos a realizar mayores esfuerzos por desarrollar viviendas de renta baja para las ciudades y priorizar la concesión de tierra a ciertos proyectos de viviendas asequibles. Diez millones de unidades de viviendas asequibles en 2011 y 36 millones de unidades de habitación hasta el 2015, de renta baja, y construir viviendas públicas de alquiler y proyectos de renovación de áreas degradadas.

\section{IMPACTO AMBIENTAL Y SOSTENIBILIDAD}

En los años 80 se inicia una reflexión sobre el desarrollo sostenible en Naciones Unidas, seguida de la Unión Europea. La publicación del informe Brundtland (WCED, 1987), también llamado Nuestro Futuro Común, ofrece una definición del desarrollo sostenible, que es referencia hasta la actualidad.

Desarrollo sostenible "el desarrollo que satisface las necesidades de la generación actual sin comprometer la capacidad de las generaciones futuras" (WCED, 1987:8). Limitaciones en el "estado de la tecnología y la organización social, o la capacidad del medio ambiente para satisfacer las necesidades actuales y las futuras (WCED, 1987:43). Incompatibilidades entre crecimiento económico y protección medioambiental y las posibilidades de mantener el crecimiento actual de los países con economías más desarrolladas. Definición utilizada en el ámbito político, institucional y científico en el debate sobre la sostenibilidad planetaria; la Cumbre de la Tierra en 1992 (López Pardo, 2015:112).

La economía china crece basándose en la industrialización, lo que provoca un enorme gasto de energía, que procede del carbón. Cada tonelada de carbón produce una tonelada de contaminación, incluyendo CO2, partículas, dióxido de azufre y mercurio. (Fort, 2014). El consumo total de energía se duplicará en 2020. China es el principal emisor de CO2 del planeta, casi duplica a Estados Unidos, el segundo de la lista. Encontrar el equilibrio entre desarrollo económico y la protección del medio ambiente, es el reto al que se enfrenta China.

En China desde 1980 la acelerada urbanización de las ciudades ha originado problemas: elevado nivel de polución y contaminación de las grandes ciudades, en especial Pekín, que cuenta con seis millones de vehículos; y la consiguiente destrucción del entorno y del medio ambiente. En China, en 2004, las enfermedades respiratorias eran la primera causa de muerte en las zonas rurales y la cuarta en las ciudades. Los chinos disponen de menos agua por habitante que la media mundial. Dos de tres ciudades tienen problemas de agua, derivados del aumento de población, la explotación del agua subterránea, la escasez de agua en 
superficie, las fugas de las cañerías y la enorme contaminación de los ríos. El 30\% de las ciudades no tienen estaciones de tratamiento de residuos urbanos.

En el verano de 2007, para superar sus problemas ambientales, China publicó su primer "Plan Nacional" para afrontar el cambio climático, aunque declaró que la preocupación por el medio ambiente no debe repercutir en el desarrollo económico. Se trataba de reducir el consumo energético en una quinta parte antes de 2010, al mismo tiempo se incrementaba la cantidad de energía renovable. Se propuso el planeamiento de más de 400 ciudades nuevas en los próximos veinte años y se espera que unos 300 millones de personas cambien su estilo de vida agrícola por un estilo industrial-urbano.

Pekín, en 2008 y para las Olimpiadas, se establecieron medidas para mejorar la calidad del aire, se cerraron plantas de electricidad y se sacaron de la ciudad industrias, se reemplazaron miles de calderas de carbón por otras de gas, y se redujeron el número de automóviles en circulación. En 2011, el número de automóviles creció de cuatro a cinco millones y las mediciones de $\mathrm{CO} 2$ se mantienen en niveles nocivos para la salud. Incidencia del cáncer de pulmón, ha crecido en $56 \%$ entre 2001 y 2010. En la capital, los hospitales están saturados de pacientes con problemas respiratorios, cardíacos o de circulación. Las autoridades sanitarias recomiendan que los mayores y los niños no salgan de casa. Treinta y seis industrias dejarán de producir y otras setenta y cinco reducirán las emisiones contaminantes en un 30\%; otras treinta y seis reducirán su producción de forma voluntaria.

El 14 de marzo de 2011 se publicó El 12 Plan Quinquenal (2011-2015), que pretende reducir la desigualdad y crear un ambiente más adecuado para el crecimiento sostenible, y conseguir una distribución más equitativa de la riqueza, aumentar el consumo doméstico, así como mejorar la infraestructura social y la red de seguridad social.

El calentamiento de 1,5 grados más que el 2014, ha provocado que los acuerdos deban ser vinculantes. China es el país que más $\mathrm{CO} 2$ emite, el aeropuerto de Pekín canceló el 25 de diciembre de 2015 cientos de vuelos por contaminación. Se cierran ciertas industrias, pero no se restringe la circulación de vehículos privados. La polución atmosférica se produce en las principales ciudades chinas, pero en invierno se acentúa debido a la calefacción pública de carbón. Científicos chinos calculan que cada año mueren 350.000 a 500.000 chinos a consecuencia de la alta contaminación ambiental. La segunda mayor economía del mundo es también la mayor consumidora de energía.

\section{ECO-CIUDADES}

El sistema integrado de residuos y energía de las eco-ciudades promete aliviar la tarea de los hogares rurales para su propia subsistencia, y también bajar las emisiones de carbono. En lugar de quemar combustible a base de carbono para el consumo de energía individual, los sistemas de biogás pueden utilizar desechos humanos, animales o de la agricultura y convertirlos en gas para calefacción y cocinar (May, 2008: 2).

\subsection{Tianjin}

Este es un importante puerto industrial cercano a Pekín, a 120 kilómetros de la capital. Puerto de las mercancías y principal centro de comercio de acero y otros metales. El proyecto para construir la Eco-ciudad Tianjin, a 45 kilometros de la antigua, en una zona pegada al mar, que sufría vertidos de las industrias contaminantes. En eco-ciudad, se utilizarán las tecnologías de ahorro energético (energía solar, eólica, el reciclaje de aguas de lluvia, el 
tratamiento de aguas residuales). Las viviendas tienen un sistema centralizado de tuberías bajo el suelo, que se calienta o enfría gracias a la energía eólica. Hay varios edificios habitados por familias que han podido comprar gracias a los precios accesibles; instalaciones comunitarias gratuitas, sistema de recogida, reciclaje y tratamiento de residuos.

En la primavera de 2012 llegan los 60 primeros habitantes, que alcanzarán los 350.000 vecinos en 2020. Habrá distintas clases sociales y pisos de varios tipos, tamaños y precios. El $60 \%$ de los desechos deberá ser reciclado; habrá disponibles 120 litros de agua al día por habitante. La lluvia se recogerá y se reciclará para riego o aguas grises - lavado y aseo-; se fomentará la vida del barrio, con colegios y hospitales en todos los vecindarios, y el trasporte rodado quedará reducido en un $90 \%$. El Gobierno chino y el de Singapur financiarán la ecociudad (Zabalbeascoa, 2012).

\subsection{Guangzhou}

Este es un centro político, económico, tecnológico, educativo y cultural situado en el sureste de la provincia de Guangdong, en el margen norte del delta del Río Perla, centro de comunicación y puerto comercial en el sur de China. Es una ciudad de siete millones de habitantes con una extensión de $3700 \mathrm{Km}^{2}$, fue una de las primeras ciudades en beneficiarse de las políticas de liberalización económica aplicada en los ochenta. Ciudad de más de 2200 años, se conocía como "Ciudad Congestionada" y "Ciudad Sucia".

En 1997, el Ayuntamiento promueve el "Plan Quinquenal de Acción", destinado a mejorar la habitabilidad de la ciudad y relanzar su atractivo como lugar de residencia y centro de negocios. El programa concluyó en 2001, con la mejora en la gestión del tráfico, la introducción de zonas verdes en la ciudad, el saneamiento, el control de la contaminación y la conservación del patrimonio natural y cultural. El plan futuro convertir Guangzhon en una ciudad ecológica para el 2010.

\subsection{Dongtan}

Las autoridades chinas proponen construir varias eco-ciudades con cero emisiones de $\mathrm{CO} 2$, con su proyecto de Eco-ciudad de Dongtan, localizada en el río Yangtze, cerca de Shanghai. Utilización de energías renovables para producción parcial de energía, el transporte limpio y la gestión de residuos, que pretende ser la primera metrópoli sostenible en 2040. El proyecto de Dongtan, en la isla de Chongming, es innovador en diseño ambiental, en una extensión de 86 kilómetros cuadrados. Una ciudad con tres villas que se irá construyendo por fases: en la primera acogerá a una población de 10.000 habitantes, en 2010. Este hito no se ha alcanzado; sin embargo, se espera que en los próximos 4 o 5 decenios esta eco-ciudad albergue una población de 500.000 habitantes. Se ha construido un túnel y un puente para unir la isla de Chongming con Shanghai, para demostrar que es posible construir una ciudad sostenible. La propiedad es de Shanghai Industrial Investment Corporation (SIIC), que ha encargado el proyecto a ARUP, una constructora internacional de planificación, ingeniería y diseño, dirigido por el arquitecto Alejandro Gutiérrez, que ha propuesto una eco-ciudad, respetuosa con su entorno medioambiental.

"Nuestro interés por involucrarnos en el proyecto radicó en la visión del cliente y en su prioridad por desarrollar una ciudad sustentable. Se proyectaría y construiría una ciudad que demostrará que es posible generar más progreso con menos víctimas. En definitiva, se trata de una ciudad en que la calidad de vida está asociada a una aproximación sustentable al desarrollo económico, social y ambiental" (Gutiérrez, 2005:1).

En la parte meridional de la isla, hay una zona de humedales, que es una reserva migratoria, la espátula menor. Se ha previsto una zona de separación entre la ciudad y los 
humedales, que en su punto más estrecho tendrá 3,5 kilómetros de anchura. Sólo se podrá construir en un $40 \%$ de la superficie terrestre de Dongtan, donde Arup se propone crear una ciudad formada por varios núcleos urbanos, conectados por carriles para bicicletas y corredores de transporte público.

Los habitantes podrán acceder a varias zonas de la ciudad en tranvía, autobuses, en bicicletas o a pie y tendrán que andar siete minutos desde cualquier parte de la ciudad hasta la parada del tranvía o autobús. Los vehículos tendrán que funcionar con combustibles renovables alternativos (incluido el hidrógeno) y habrá un acceso fácil a varias modalidades de trasporte público, incluidos autobuses de pila de combustible y taxis acuáticos con energía solar en canales y lagos. Una combinación de carriles para bicicletas y zonas peatonales reducirá el ruido y la contaminación atmosférica; los visitantes aparcarán sus coches a las afuera de la ciudad y usarán transporte público para acceder a la misma.

Se utilizarán diferentes fuentes de renovables, como turbinas eólicas, biocombustible y materias orgánicas recicladas. Se emplearan tecnologías de construcción innovadoras para los edificios, que reduzcan las necesidades energéticas hasta un $70 \%$, instalando techos verdes en los edificios para mejorar el aislamiento y la filtración de aguas. Arup espera crear una ciudad con huella de carbono cero, pero China ha abandonado el proyecto, al menos temporalmente, sólo se ha construido el túnel y el puente, debido a sus altos costes imposibles en medio de la crisis económica. Fracaso de Dongtan, postulada como la ciudad solución para el futuro chino y el lider del partido comunista de la ciudad, Chen Liangyu terminaba encarcelado por fraude.

\subsection{Hangzhou}

Esta es una ciudad sostenible, a una hora de tren, al sur de Shanghai. El corazón de la ciudad es un lago Oeste (Xihu) rodeado de bosques y jardines circundantes ocupa alrededor de 60 kilómetros cuadrados. Contará con espacios verdes públicos $-10 \mathrm{~s} \mathrm{~m}^{2}$ de zona verde por habitante están por debajo de lo recomendado por la OMS-. El $80 \%$ de la electricidad será generada por energía eólica y solar, y el $60 \%$ de los desechos serán reciclados. Se creará un sistema de tren ligero, el $90 \%$ de los viajes se realizará a pie, en bicicleta o transporte público. Un aspecto positivo de esta nueva eco-ciudad es que se plantea el desarrollo económico junto al social (González, 2012:1).

\subsection{Model City}

Se encuentra en el pueblo de Huangbaiyu, es un proyecto de William McDonough (Tokio 1951), una eco-ciudad de dos millones de habitantes, 100\% sostenible. Fundador del estudio McDonough + Partners. Los sistemas de biogás, junto a una infraestructura de aguas residuales y electricidad solar, fueron el plan para reconstruir el pueblo de Huangbaiyu. Los desechos se usarían como combustible para una planta de biogás, que abastecería de energía a la comunidad; la energía provendría del sol, el agua corriente entraría a la casa por vez primera, y las viviendas serían construidas con materiales fáciles de retornar a la tierra o reciclar. El arquitecto asumió el reto de proyectar viviendas sustentables para ese valle rural $\mathrm{y}$, siguiendo el drenaje de las cuencas acuíferas a fin de averiguar en qué lugar del valle era posible realizar un proyecto sostenible. (May, 2008: 2). La puesta en marcha en Huangbaiyu de una planta de biogás hundiría a la comunidad local, aunque cumpliría con los objetivos de la sustentabilidad global: bajar las emisiones de carbono (los tallos de maíz fueron confundidos con desecho por el equipo encargado de la empresa, y en el invierno son la principal fuente de alimentación de las ovejas cashemire - el producto comercial más preciado de la zona y las familias se quedaban sin ingresos - (May, 2008: 3). 


\subsection{Chengdu Tianfu Great City}

El estudio de arquitectura estadounidense Adrian Smith y Gordon Gill ha propuesto para Chengdu Tianfu Great City, una ciudad satélite, que con sólo 1,3 $\mathrm{km}^{2}$ servirá para alojar 80.000 habitantes. El proyecto patrocinado por Beijing Vantone Real Estate Co. Ltd., ha previsto hacer de esta Gran Ciudad un espacio sostenible, capaz de un $48 \%$ menos de energía, un $58 \%$ menos de agua, reducir la basura en un $89 \%$, y generar un $60 \%$ menos de emisiones contaminantes. Se utilizará energía renovable y se evitará el uso del automóvil. Pensada para recorrerla a pie en 15 minutos, con trasporte público que la conecta con localidades vecinas, En una ubicación de valles y cursos de agua, un $15 \%$ de la superficie se destinará a espacios verdes, un $60 \%$ para construcción y un $25 \%$ para carreteras y calles peatonales. Se estima que se finalizará en 2020 y el objetivo es poder hacer replicas en otras zonas del país (Smith \& Gill, 2012).

\section{CONCLUSIONES}

En China, hay una dependencia del carbón: casi el 70\% de la energía a nivel nacional. El plan de Beijing para tener aire puro para 2030, incluye cierre de fábricas y reducción del uso de carbón. El presidente Xi Jinping ha dicho que la lucha contra la contaminación es una prioridad. El Instituto Municipal para la Protección Ambiental propone reducir a menos de 35 microgramos por metro cúbico en 2030.

Crisis ambiental causada por la energía sucia. La evolución del tejido urbano es una consecuencia de las decisiones que adoptan los agentes urbanos: propietarios, promotores, constructores y gestores políticos. Necesidad de planificar respetando la naturaleza y superar el espacio urbano y suburbano para realizar una ordenación territorial. Administraciones públicas y gobiernos tienen que implicarse. Las energías alternativas en la actualidad más caras, pero están reduciendo precios. El cambio en las eléctricas está en marcha y las energías limpias producen más empleo. El reto planteado en China en la actualidad, es cómo crecer con equilibrio social, con control de consumo energético y de recursos naturales, minimizando el impacto ambiental.

En los aproximadamente treinta años de crecimiento económico de China han aportado beneficios, pero también problemas que afectan a la calidad de vida de la nueva sociedad urbana, Presión sobre los recursos, en particular sobre el agua y la energía. Los promotores evitan los edificios sustentables por sus altos costes. En un país de rápido desarrollo económico y número uno mundial en volumen anual de construcción, las medidas llevadas a cabo por el gobierno chino, los promotores y las compañías privadas, no potencian la construcción de edificios sostenibles.

China se enfrenta al reto de reducir la contaminación atmosférica. Ha creado grandes metrópolis que son altamente contaminantes y destructivas. Es necesaria una menor contaminación, eficiente uso de la tierra y reducción de las causas que generan el calentamiento global. El gobierno ha reducido en un 5\% el consumo de carbón para 2017, para frenar la contaminación del aire. Invertirá alrededor de 300.000 millones de dólares para combatir la contaminación. El 36,7 \% es destinado a la industria de "limpieza del aire", otro $28,2 \%$ al impulso de las fuentes de energía renovable; el resto se empleará para mejorar las calidades de los motores de vehículos. Plan de mejora medioambiental para el periodo 20132017.

El urbanismo y la arquitectura, en el siglo XXI, han estado condicionadas al desarrollo económico especulativo y desordenado, a pesar de algunos arquitectos y urbanistas que han 
tenido en cuenta la dimensión social de la arquitectura, su incidencia en la calidad de vida de los ciudadanos, así como en la repercusión en las generaciones futuras.

\section{BIBLIOGRAFÍA}

AMIANO, I. (2009). "Arquitectura china”, en: http://www.isabelamiano.com.ar/wpcontent/uploads/ 2009/03/china.pdf .

ANGUIANO DE MIGUEL, A. (2009). "Museos Centros de Arte Contemporáneo de la Posmodernidad", en Gallego Trijueque, S. y Gómez Escarda, M. (Coords.) Igualdad, desarrollo y sostenibilidad. Toledo: ACMS, pp. 219-236.

ANGUIANO DE MIGUEL, A. (2015). "Desarrollo urbano en países emergentes", en González García, E.; García Muñiz, A.; García Sansano, J. e Iglesias Villalobos, L. (Coords.) Mundos emergentes: cambios, conflictos y expectativas. Toledo: ACMS, pp. 238-248.

BLÁZQUEZ, P. (2011). "El club de las ciudades habitables", en: www.ethic.es/2011/12.

BRUNET ICART, I. (2016). "La construcción del capitalismo financiarizado", XIV Premio "Fermín Caballero" de ensayo breve en ciencias sociales- 2015. Barajas de Melo: ACMS, pp.51-72.

CAPEL, H. (2013). La morfología de las ciudades. III. Agentes urbanos y mercado inmobiliario. Barcelona: Ediciones del Serbal.

CERVERA, J. (2013). “Una Seseña junto a Shanghai”, en: blog.rtve.es/retiano/2013/09.

CERVERA, R. et al., (n.d.). "Luces y sombras de la expansión urbana china". Informe colectivo del grupo Barcelona Shanghai Wome Bridge, en: www.catedrachina.com .

FORT, P. (2014). "El plan de Beijing para tener aire puro en 2030", en: www.chinafiles.com /es/link/39850.

FOSTER, N. (2013). Catalogue Foster and Partners. Munich: Prestel.

FOSTER, R. (2013). "Norman Foster. In the 21st Century". AV Arquitectura Viva. Monografías, 163-164: 96-99 y 286-293.

FUTAGAWA, Y. (2014). Jean Nouvel. Recent Project. Tokio: A.D.A.

GÓMEZ MENDOZA, J. (2012). "La urbanización china como motor de crecimiento económico. Herencias socialistas y liderazgo urbano en la atracción del capital”, en: http://goo.gl/ZWe7bS.

GONZÁLEZ, V. (2012). "Conoce más sobre Tianjin, la futura eco-ciudad en China", en:

https://www.veoverde.com/2012/05.

GUTIÉRREZ, A. (2005). "Nueva ciudad de Dongtan”, en: www.scielo.cl/pdf/arq/n60/art08.

HADID, Z. (2011). "Opera de Guangzhou”, en: www.plataformaarquitectura.cl/cl/626383.

HERZOG \& DE MEURON (2006). "Campus Tree Village", Herzog \& de Meuron 2002-2006.

Basel, Schaulger, pp.166-173.

HERZOG \& DE MEURON (2006). "2002/2006 Hergoz \& de Meuron”. El Croquis Monografías, 129/130: 348-373.

KOOLHAAS, R. (2007). “AMO/OMA. Rem Koolhaas. Teoría y práctica. 1996-2007”. El Croquis, 134-135: 30-43 y 178-187.

KOOLHAAS, R. (2015). "Rem Koolhaas OMA/AMO 2000-2015”, AV Monografías, 178-179: 112$123 ; 130-137 ; 172-177$ y $186-189$.

LALUETA, I. (2013). “Aeropuerto Internacionl Shenzhen Bao”, en: www.metalocus.es

LALUETA, I. (2014). "Comienza la construcción del Complejo Cultural de Mecanoo en Shenzhen”, en: www.metalocus.es

LASERNA, D. (2014). "Hangzhou y la magia verde", en: www.china-files.com/es/link/42540.

LÓPEZ PARDO, I. (2015). "Sobre el desarrollo sostenible y la sostenibilidad: conceptualización y crítica". Barataria. Revista Castellano-Manchega de Ciencias Sociales, 20:111-128.

LÓPEZ-PUJOL, J. (2008). "Impactos sobre la biodiversidad del embalse de las Tres Gargantas en China". Ecosistemas. Revista Científica y Técnica de Ecología y Medio Ambiente, pp.134-145.

MAY, S, (2008). "Crisis ecológica y eco-aldeas y eco-ciudades en China", en: www.rebelion.org.no ticias.php?id=77251. 
MIGUEL, J. M. de (2014). "Debate sobre el incremento de la desigualdad: en torno a Piketty", en García Sansano, J; González García, E.; Lago Morales, I. y Rubio Sánchez, R. (Coords.) Tiempos oscuros, décadas sin nombre. Toledo: ACMS, pp. 344-371.

NAREDO, J. M. (2004). "Diagnóstico sobre sostenibilidad: la especie humana como patología terrestre", en A. Hernández Aja (Ed. y Coord.) Sostenibilidad en el proyecto arquitectónico y urbanístico. IAU+S. Madrid, ETSAM, Universidad Politécnica.

NEGRE, M. I. (2007). "La presa de Tres Gargantas: una obra monumental", en Observatorio de la Economía y la Sociedad de China, no 2, marzo 2007, en: http://www.eumed.net/rev/china.

NOUVEL, J. (2014). Musée national des arts de la Chine (Namoc). Architecture d' Aujourd'hui, 403: 16-17.

OLLERO, D. J. (2015). "China compra edificios fantasma para aliviar su burbuja inmobiliaria". El Mundo, 07/08/2015, en: http://goo.gl/yGIjw3 .

PAULINE, J. (2014). "Centro Nacional de Natación, Pekín". AC/CA Arquitectura contemporánea, en: compo3t.blogspot.com.es/2014/11/ptw.

PLAN de acción para Guangzhou sostenible, China, en: habitat.a.g.upm.es/dubai/02/bp276html.

SÁNCHEZ MOSQUETE, J. (2010). "Megaconstrucciones: Aeropuerto de Hong Kohn, en: www.mosingenieros.com/2010/01.

SHU, W. (2012). "WangShu: Académico, artesano y arquitecto", en: www.realestatemarket.com/ articu los/arquitectura/11571.

SMITH, A. y GILL, G. (2012). "Chengdu Tianfu Great City”, en: smithgill,com/news/great_city_ press_release.

ZABALBEASCOA, A. (2009). "Siete arquitectos que cuidan el Planeta". Arquitectura y Diseño, 10: 106.

\section{Breve currículo:}

\section{Aida Anguiano de Miguel}

Licenciada en Historia y Doctora en Arte por la Universidad Complutense de Madrid. Profesora del Departamento de Historia del Arte de la Universidad Complutense de Madrid, y Profesora del Departamento de Ciencias Sociales de la Universidad Rey Juan Carlos. Desde 2005 pertenece a la Asociación Castellano-Manchega de Sociología. En la actualidad, retirada de la docencia, continúa con sus líneas de Investigación: Urbanismo, Arquitectura y Artes Plásticas Contemporáneas, desde un método sociológico. 